\title{
Synthesis of Peres and R Logic Circuits in Nanoscopic Scale
}

\author{
Md. Abdullah-Al-Shafi \\ Dept. of ICT \\ Mawlana Bhashani Science \\ and Technology University, \\ Tangail, Bangladesh
}

\begin{abstract}
Traditional lithography based VLSI architecture expanded to optimize higher scale integration and low powered computing of semiconductor components. This tend encounter severe challenges of short channel issue, tunneling and variation of doping. Quantum Dot Cellular Automata (QCA) is an esteemed nano-scale technology and a better substitute of Complementary Metal Oxide Semiconductor (CMOS) for it's transformation technique and computation method. Information-lossless or reversible logic circuits has divers precise utilization in communications, digital signal processing (DSP), computer graphics also in quantum computation. This paper presents the systematic model of Peres gate (PG) and R gate in QCA and CMOS. To simulate and verify the proposed design QCADesigner and Microwindlite, widely used simulation tools are used. Those proposed design has a promising future and can be used in modeling complex computing structure and nano-scale based low power consumption information processing structure.
\end{abstract}

\section{Keywords}

QCA, Peres gate, R gate, QCADesigner, MICROWIND.

\section{INTRODUCTION}

Nanotech has been the focus of a comprehensive analysis to repudiate the limitations of CMOS and imparts a unique magnitude for computing. Today it is admitted that next two decades, semiconductor production will have to preface applying new nanoelectronic materials [1]. Quantum dot Cellular Automata is a favorable technology for forthcoming ICs [2,3] and a better substitute of complementary metal oxide semiconductor (CMOS) technology [4]. The reversible circuits compose the decisive architecture of quantum computers and typically used in power deprecation having the influence in emerging technologies as low power CMOS design, quantum computing, computer graphics, cryptography, communication, and optical computing $[5,6,7$, $8]$. In the early $1960 \mathrm{~s}, \mathrm{R}$. Landauer manifested that high technology circuits and systems constructed using irreversible logic circuit outcomes in energy dissipation due to information slump. Landauer's principle demonstrates that the loss of one bit of information lost, will depletes $k \mathrm{~T} \ln (2)$ joules of energy, where $k$ is the Boltzmann's constant and T is performing temperature in Kelvin [9]. Later Bennett reveals that a zero power dissipation in logic circuits is conceivable, if it is contrived of reversible circuits [10]. Reversible circuits can be viewed as exclusive instance of quantum circuits as quantum expansion must be reversible [11]. In the area of quantum computing there are divers propositions on reversible circuit design like Feynman Gate [12], Toffili Gate [13], Fredkin Gate [14], NFT Gate [15] but very less of them are designed in a competent means in QCA [16, 17, 18]. The paper presents the architecture of Peres gate (PG) and R gate in QCA which is effectual than earlier designs and also presents the CMOS design of each individual layout.

\section{BASICS OF QCA}

The basic building segment of QCA is QCA cell and the cell is composed of four quantum dots located at the edges of the squared cell and two loose electrons. Depending on the location of the electrons, QCA cell has two form of polarization $[19,20]$. The polarization of electrons are defined as $\mathrm{P}=+1$ for logic ' 1 ' and $\mathrm{P}=-1$ for logic ' 0 ' shown in figure 1.

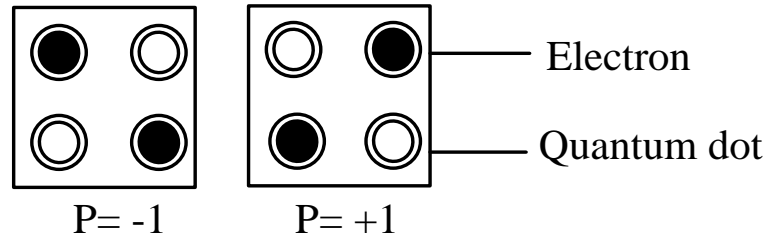

Fig 1: Basic structure of a QCA cell

The elemental entity of QCA based layout is QCA wire, majority gate and inverter [20]. QCA wire is a cluster of cells which regulated one by one and the polarization of each cell is explicitly influenced by the polarization of its neighboring cells accordingly electrostatic force. QCA wires can be either contrived up of $45^{\circ}$ cells or $90^{\circ}$ cells shown in figure 2 . Basically QCA wires used to proliferate binary data from one end to another.

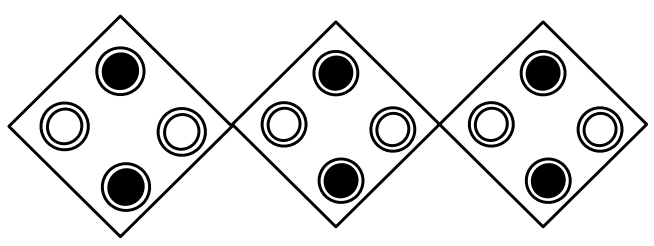

(a)

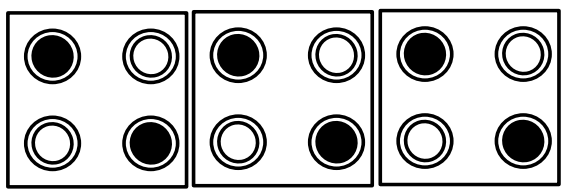

(b)

Fig 2: QCA wire (a) $45^{\circ}$ (b) $90^{\circ}$ 
Roughly a majority voter composed of five cells and three of them are inputs, one output and one is middle cell. The center cell named device cell by reason of its action, switches to primary polarization [20]. Majority Voter can be expressed as $\mathrm{MV}(\mathrm{A}, \mathrm{B}, \mathrm{C})=\mathrm{AB}+\mathrm{BC}+\mathrm{AC}$. Using majority gate two primitive gates "AND" and "OR" can be implemented by assign one of the input fixed to 1 or 0 value. Figure 3 shows when $\mathrm{C}=1$ the output is $\mathrm{A}+\mathrm{B}$ which means $\mathrm{OR}$ function and when $\mathrm{C}=0$ the output is $\mathrm{AB}$ that indicates the $\mathrm{AND}$ function.
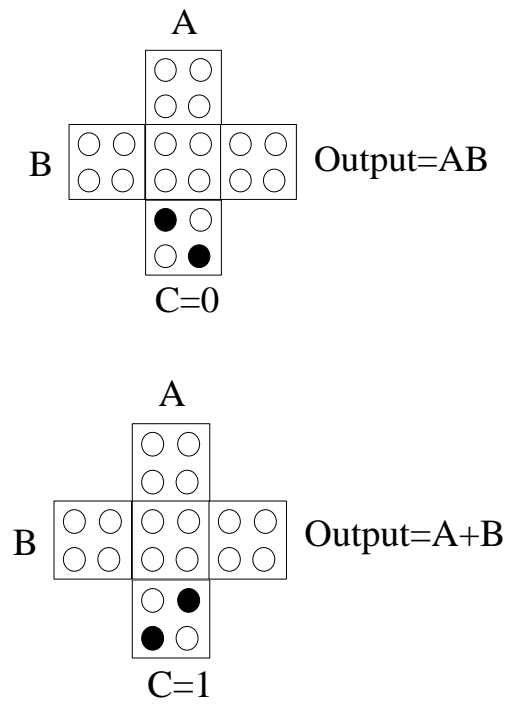

Fig 3: Majority Voter as AND gate $(C=0)$ and $O R$ gate $(\mathbf{C}=\mathbf{1})$

QCA inverter returns the reverse value of input. This inverter is built of four QCA wires or seven cell. The input polarization is slit into two polarizations and finally, two wires join and make the opposite polarization shown in figure 4.

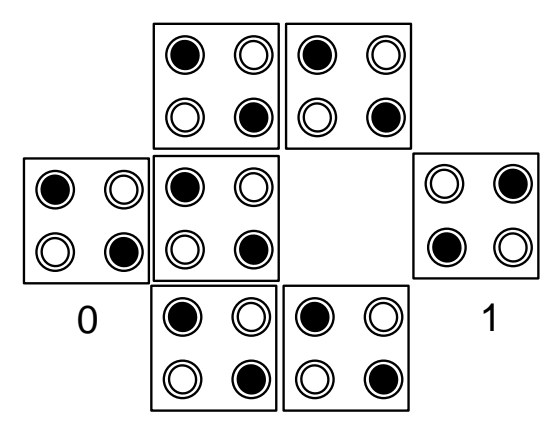

Fig 4: QCA seven cell inverter

\section{PROPOSED CIRCUIT AND PRESENTATION}

A circuit is called reversible if there is a one to one correspondence between its input and output positions or a gate is called reversible if the function it computes is bijective. A reversible circuit should be formed using nominal number of reversible gates. This section presents the proposed structures of Peres and R gate.

\subsection{Peres Gate}

Peres gate (PG) is a 3-input, 3-output, reversible gate [21] where the input is $\mathrm{I}(\mathrm{A}, \mathrm{B}, \mathrm{C})$ and corresponding output is $\mathrm{O}$ $(\mathrm{P}, \mathrm{Q}, \mathrm{R})$. The outputs are defined by $\mathrm{P}=\mathrm{A}, \mathrm{Q}=\mathrm{A} \oplus \mathrm{B}$ and $\mathrm{R}=\mathrm{AB} \oplus \mathrm{C}$. The quantum cost of Peres gate is 4. Figure 5(a) presents the block diagram of Peres gate and (b) shows the QCA representation.

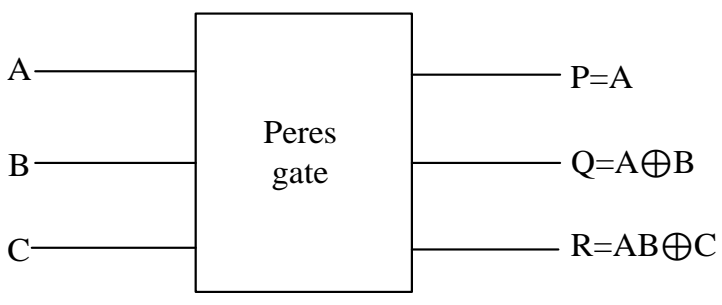

(a)

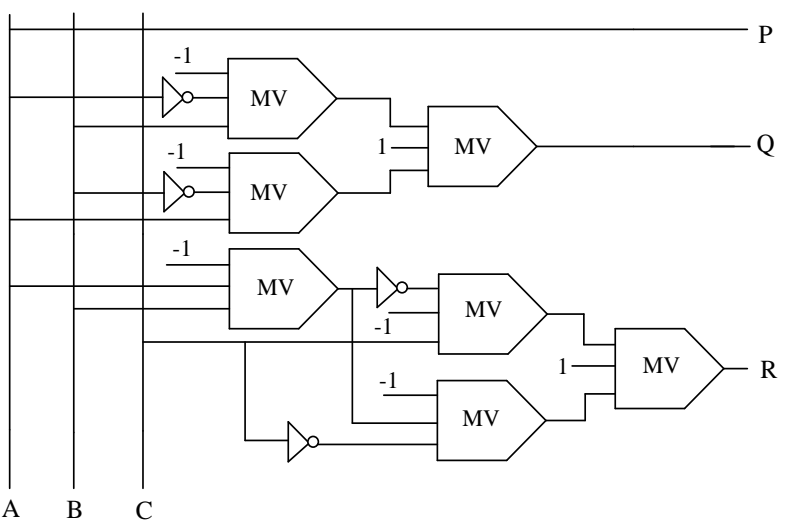

(b)

Fig 5: Block diagram of (a) Peres gate (b) proposed diagram of Peres gate in QCA

Table 1. Truth table presentation of peres gate

\begin{tabular}{|c|c|c|c|c|c|}
\hline \multicolumn{3}{|c|}{ Input } & \multicolumn{3}{c|}{ Output } \\
\hline $\mathbf{A}$ & $\mathbf{B}$ & $\mathbf{C}$ & $\mathbf{P}$ & $\mathbf{Q}$ & $\mathbf{R}$ \\
\hline 0 & 0 & 0 & 0 & 0 & 0 \\
\hline 0 & 0 & 1 & 0 & 0 & 1 \\
\hline 0 & 1 & 0 & 0 & 1 & 0 \\
\hline 0 & 1 & 1 & 0 & 1 & 1 \\
\hline 1 & 0 & 0 & 1 & 1 & 0 \\
\hline 1 & 0 & 1 & 1 & 1 & 1 \\
\hline 1 & 1 & 0 & 1 & 0 & 1 \\
\hline 1 & 1 & 1 & 1 & 0 & 0 \\
\hline
\end{tabular}

\subsection{R Gate}

The $\mathrm{R}$ gate is a $3 \times 3$ reversible gate [22]. Figure 6 shows the block diagram and QCA diagram of $\mathrm{R}$ gate. The input vector defined as $\mathrm{I}(\mathrm{L}, \mathrm{M}, \mathrm{N})$ and the output vector is $\mathrm{O}(\mathrm{X}, \mathrm{Y}, \mathrm{Z})$. The outputs are followed by $\mathrm{X}=\mathrm{L} \oplus \mathrm{M} ; \mathrm{Y}=\mathrm{L}$ and $\mathrm{Z}=\mathrm{LM} \oplus \mathrm{N}^{\prime}$. 


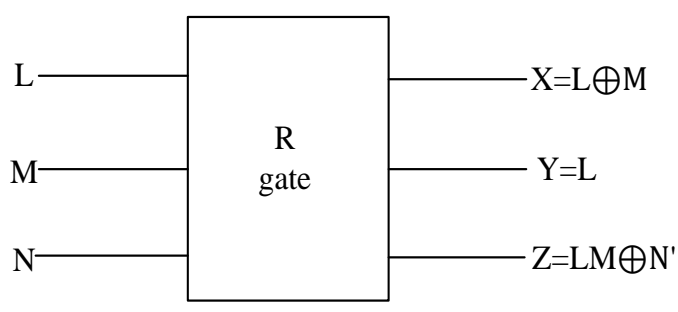

(a)

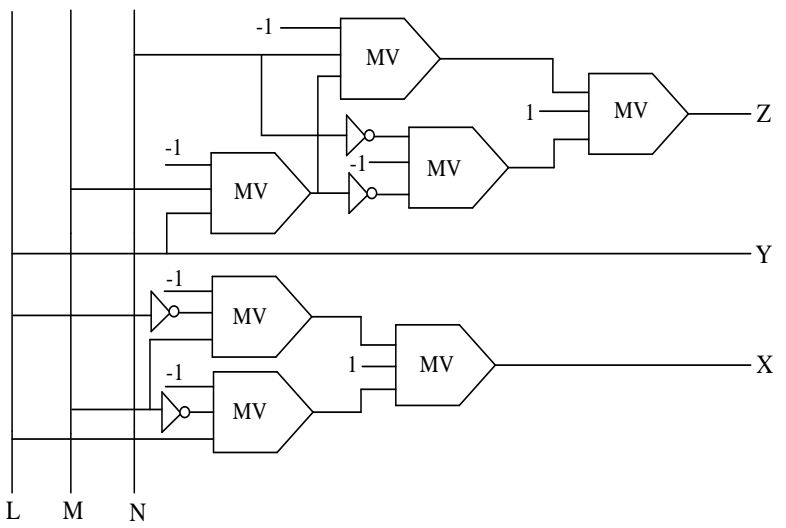

(b)

Fig 6: Block diagram of (a) $R$ gate (b) proposed diagram of $R$ gate in QCA

Table 2. Truth table presentation of $R$ gate

\begin{tabular}{|c|c|c|c|c|c|}
\hline \multicolumn{3}{|c|}{ Input } & \multicolumn{3}{c|}{ Output } \\
\hline $\mathbf{L}$ & $\mathbf{M}$ & $\mathbf{N}$ & $\mathbf{X}$ & $\mathbf{Y}$ & $\mathbf{Z}$ \\
\hline 0 & 0 & 0 & 0 & 0 & 1 \\
\hline 0 & 0 & 1 & 0 & 0 & 0 \\
\hline 0 & 1 & 0 & 1 & 0 & 1 \\
\hline 0 & 1 & 1 & 1 & 0 & 0 \\
\hline 1 & 0 & 0 & 1 & 1 & 1 \\
\hline 1 & 0 & 1 & 1 & 1 & 0 \\
\hline 1 & 1 & 0 & 0 & 1 & 0 \\
\hline 1 & 1 & 1 & 0 & 1 & 1 \\
\hline
\end{tabular}

\section{TECHNICAL IMPLEMENTATION OF} PERES AND R CIRCUIT

Various proximate simulators such as the bistable simulation and nonlinear approximation are employed for testing the design level. But these approaches are iterative so it cannot produce the certain products. Finally QCA Designer ver. 2.0.3 is picked and this simulation engine is explained [23]. The definiteness of the proposed circuits are estimated by the simulation tool of QCA Designer ver. 2.0.3 [24]. The technical presentation are shown below.

\subsection{Peres Gate}

The quantum implementation of peres circuit has been urged by various authors [25] which is shown in figure 7(a) and figure 7(b) delineates the proposed peres circuit

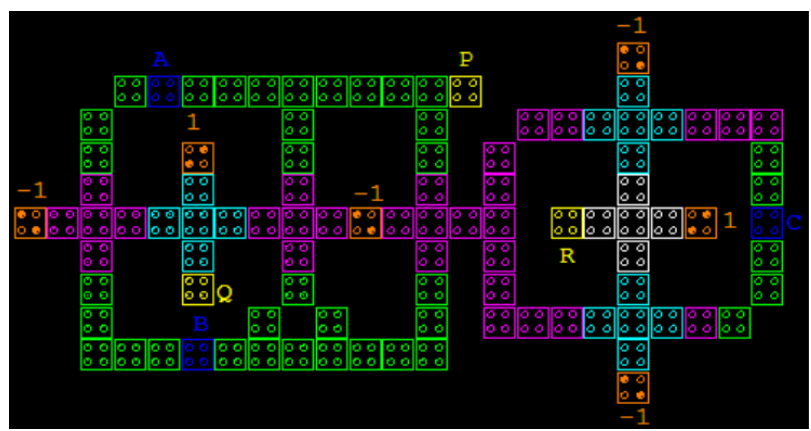

(a)

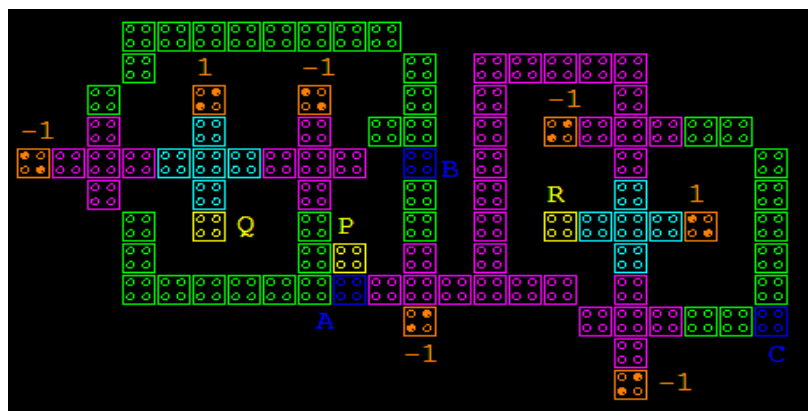

(b)

Fig 7: QCA simulated circuit design of (a) Peres circuit (b) proposed Peres circuit

\subsection{R Gate}

The QCA implementation of R circuit is shown in figure 8(a) and has been proposed by distinct authors [26]. The proposed $\mathrm{R}$ circuit is shown in Figure 8(b).

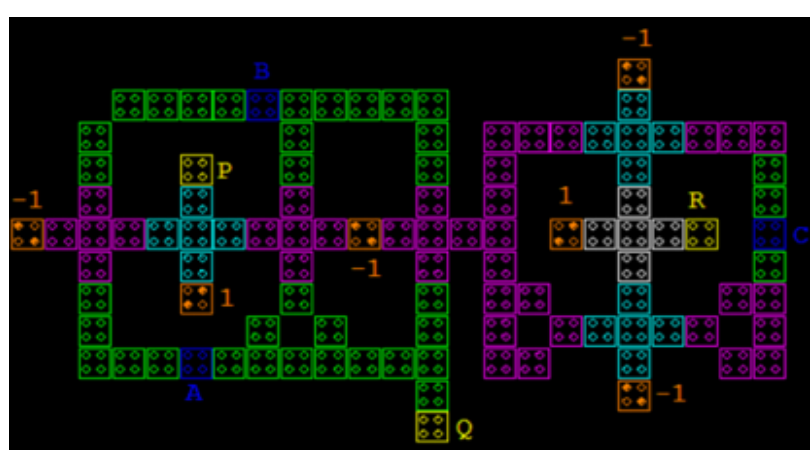

(a)

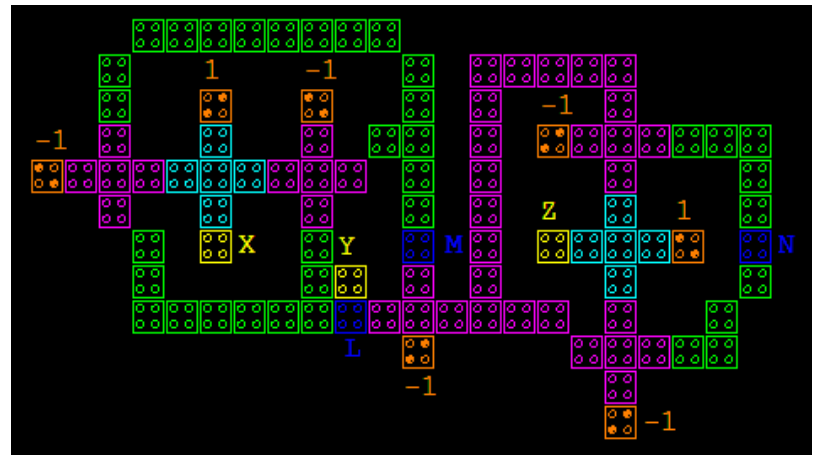

(b)

Fig 8: QCA simulated circuit design of (a) $R$ circuit (b) proposed $\mathbf{R}$ circuit 


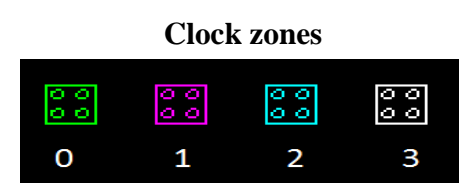

For simulation and composition the proposed circuits in CMOS, MICROWIND [27] a PC tool for CMOS design is employed. This engine is very user-friendly to model and find out the covered space of any logic circuit as shown in figure 9 where (a) prescribed the peres circuit and (b) specify the R circuit.

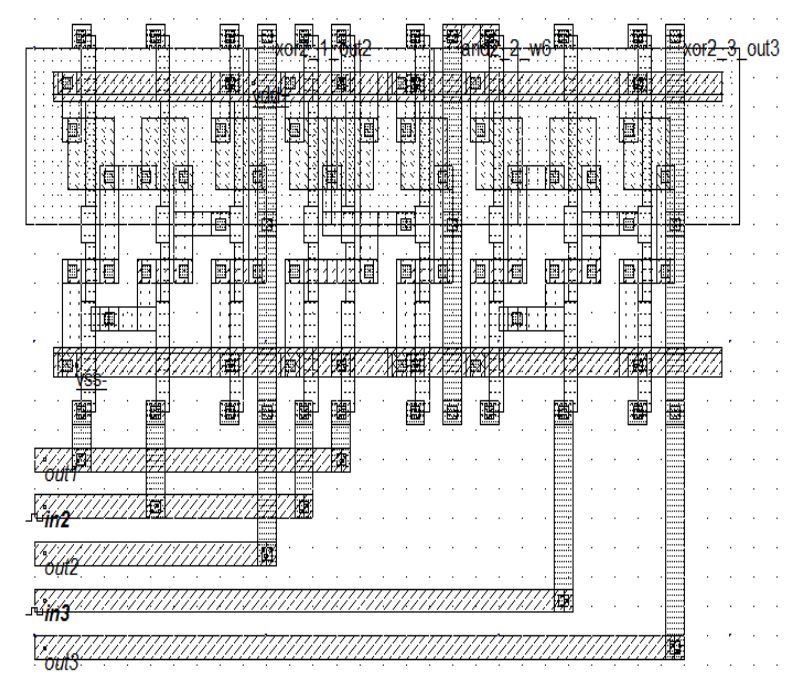

(a)

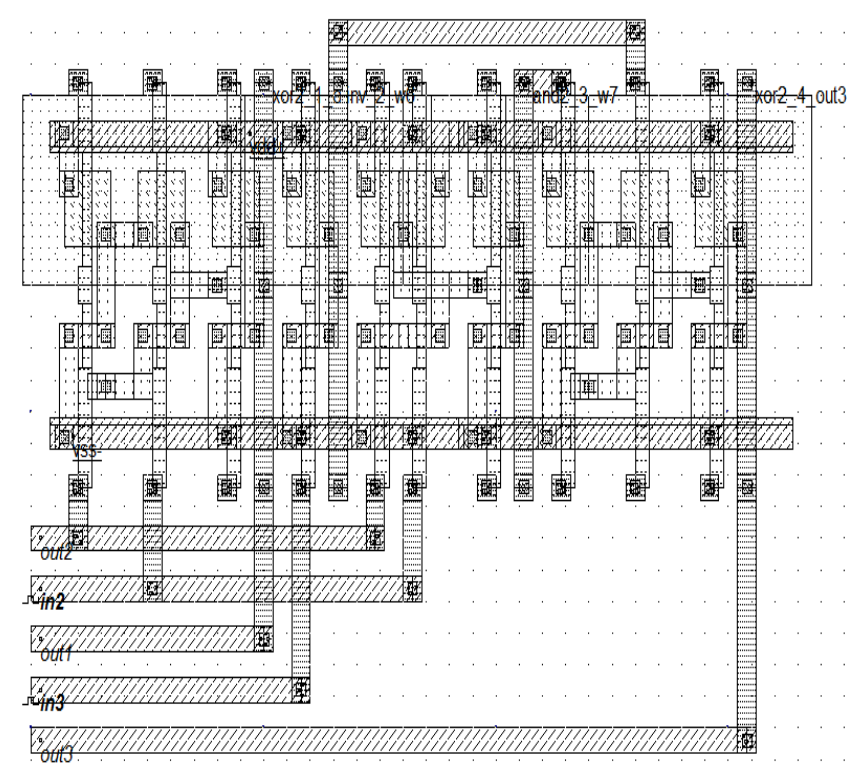

(b)

Fig 9: Simulated circuit design of (a) Peres gate (b) R gate

\section{SIMULATION AND RESULT \\ ANALYSIS}

The urged logic circuits were functionally simulated using QCA Designer ver. 2.0.3. The successive criterions of the bistable approximation and coherence vector is used which are the default rates in QCADesigner. These parameters are shown in table 3 .
Table 3. Criterions of Bistable Approximation and Coherence Vector

\begin{tabular}{|l|l|}
\hline cell size & $18 \mathrm{~nm}$ \\
\hline dot diameter & 5.000 \\
\hline number of samples & 12800 \\
\hline relaxation time & $1.000000 \mathrm{e}-15 \mathrm{~s}$ \\
\hline radius of effect & $65.000000 \mathrm{~nm}$ \\
\hline time step & $1.000000 \mathrm{e}-16 \mathrm{~s}$ \\
\hline convergence tolerance & 0.001000 \\
\hline relative permittivity & 12.900000 \\
\hline clock shift & 0 \\
\hline clock amplitude factor & 2.000000 \\
\hline layer separation & 11.500000 \\
\hline clock high & $9.800000 \mathrm{e}-022 \mathrm{~J}$ \\
\hline clock low & $3.800000 \mathrm{e}-023 \mathrm{~J}$ \\
\hline upper threshold [1] & 0.500 \\
\hline lower threshold [0] & -0.500 \\
\hline total simulation time & $7.000000 \mathrm{e}-11 \mathrm{~s}$ \\
\hline maximum iterations per sample & 100 \\
\hline
\end{tabular}

The simulated outcomes of the proposed circuits are shown below.

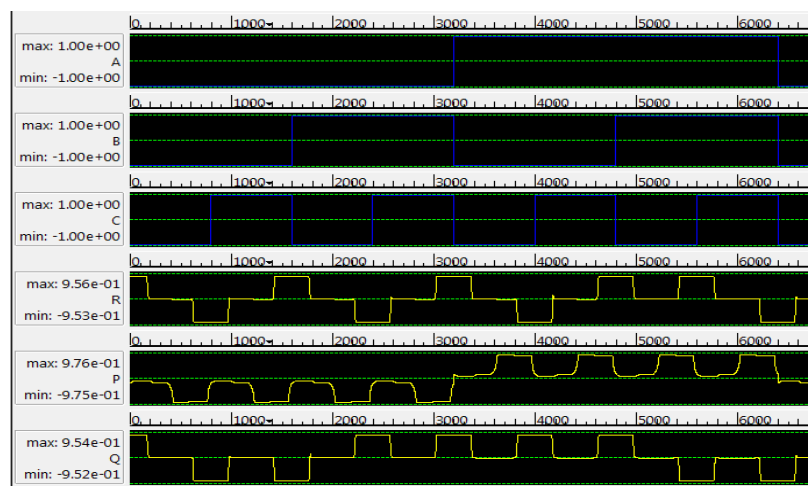

(a)

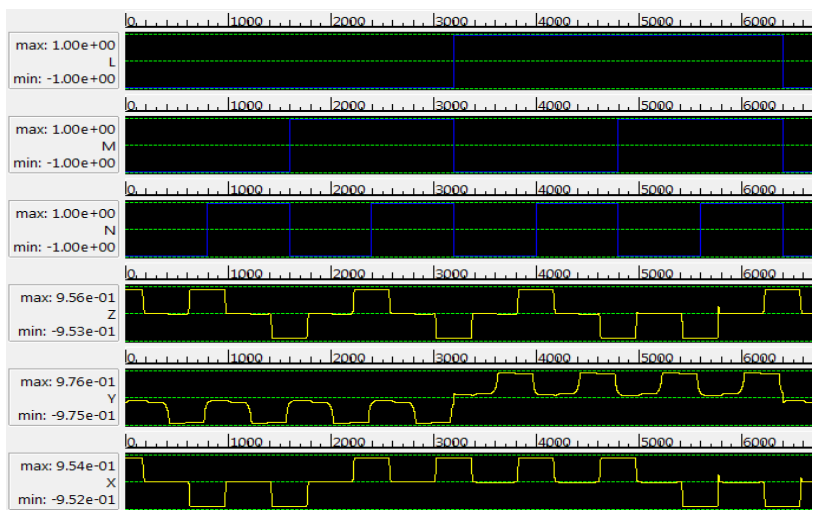

(b)

Fig 10: Simulated results in QCADesigner (a) Peres circuit (b) $\mathbf{R}$ circuit 
Table 4. Performance Analysis of Proposed Circuits

\begin{tabular}{|c|c|c|c|c|}
\hline Criterion & $\begin{array}{c}\text { Peres } \\
\text { circuit }\end{array}$ & $\begin{array}{c}\text { Proposed } \\
\text { Peres } \\
\text { circuit }\end{array}$ & $\begin{array}{c}\text { R } \\
\text { circuit }\end{array}$ & $\begin{array}{c}\text { Proposed } \\
\text { R circuit }\end{array}$ \\
\hline Cell number & 99 & 96 & 105 & 96 \\
\hline $\begin{array}{c}\text { Clock delay } \\
\text { (Clock cycle) }\end{array}$ & 1.00 & 0.75 & 0.75 & 0.50 \\
\hline $\begin{array}{c}\text { Area in QCA } \\
\left(\mu \mathrm{m}^{2}\right)\end{array}$ & 0.1008 & 0.105 & 0.126 & 0.109 \\
\hline $\begin{array}{c}\text { Area in } \\
\text { CMOS }\left(\mu \mathrm{m}^{2}\right)\end{array}$ & \multicolumn{2}{|c|}{59.8} & & 71.4 \\
\hline $\begin{array}{c}\text { Improvement } \\
\text { (in times })\end{array}$ & \multicolumn{2}{|c|}{570} & \multicolumn{2}{|c|}{655} \\
\hline
\end{tabular}

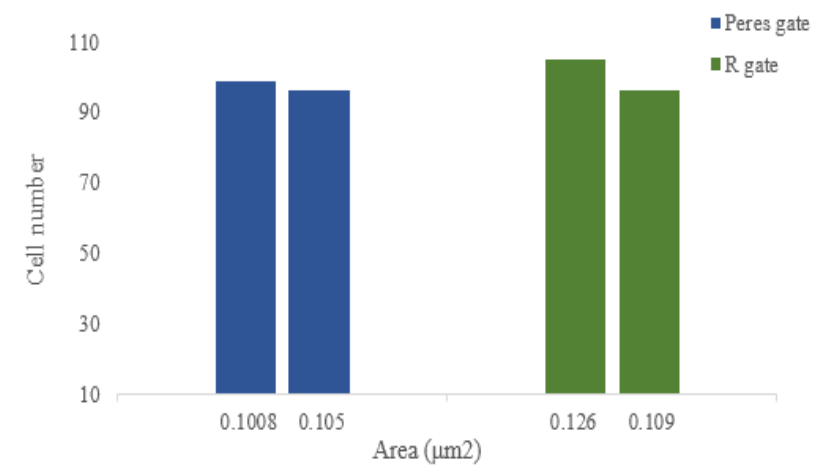

Fig 11: Comparative figures for cell and area of proposed Peres and $\mathbf{R}$ circuit

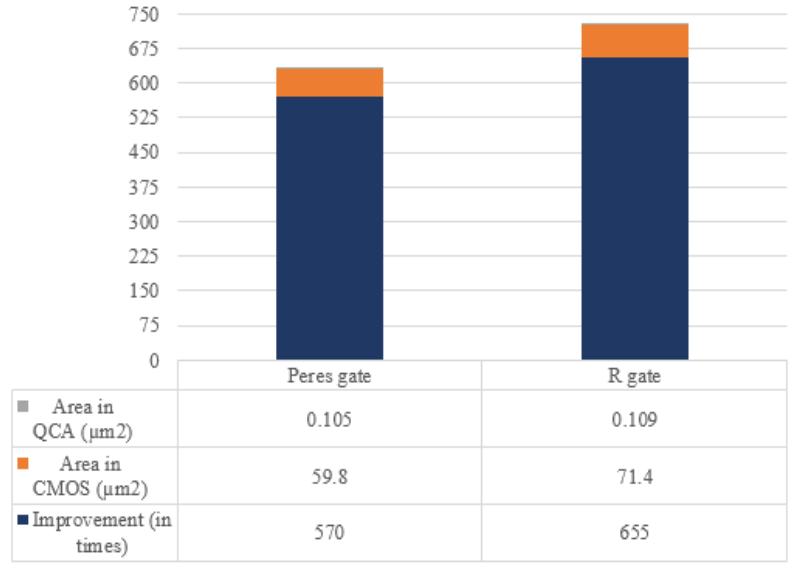

Fig 12: Comparative figures for area of QCA and CMOS with improvement

\section{CONCLUSION}

This paper analyzes an innovative design of Peres and $\mathrm{R}$ circuit and approved in terms of area and cell complication. Also the design of QCA based circuits with smaller feature size and ultralow power consumption than typical CMOS have been conferred in this paper. The simulation sequel show that the proposed design perform well. Hence, it consummates that the urged layout could be an auspicious step for future computing like quantum computers and ultralow power design in nanotechnology.
The forthcoming work can be extended as to design systems to handle larger and more general circuits with the inevitable objective of synthesizing quantum circuits.

\section{REFERENCES}

[1] Antonelli, D. A. et al, June 2004. Quantum-dot cellular automata (QCA) circuit partitioning: problem modeling and solutions. In Proceedings of the 41st annual Design Automation Conference, ACM, pp. 363-368.

[2] C. S. Lent, P. D. Tougaw and W. Porod "Quantum Cellular Automata: The physics of computing with quantum dot molecules", Physics and Computation, pp. 5-13, 17-20 Nov 1994.

[3] C. S. Lent and P.D. Tougaw "Device architecture for computing with quantum dots", Proceedings of the IEEE. Vol.85, pp.541-557, 1997

[4] H. Cho and E. E. Swartzlander "Adder and multiplier design in quantum-dot cellular automata", IEEE Transactions on Computers, Vol.58, No. 6, pp. 721-727, 2009.

[5] Al-Rabadi, Anas N "Reversible logic synthesis: From fundamentals to quantum computing", Springer, 2004.

[6] Thapliyal, H. and Ranganathan, N. 2008. Testable reversible latches for molecular QCA, In Nanotechnology, NANO '08. 8th IEEE Conference, pp. 699-702.

[7] X. Ma, J. Huang, C. Metra and F. Lombardi "Reversible gates and testability of one dimensional arrays of molecular QCA", J. Electronic Testing, Vol. 24, 297311, 2008.

[8] H. Thapliyal and N. Ranganathan "Reversible logic-based concurrently testable latches for molecular QCA", Nanotechnology, IEEE Transactions on, Vol. 9, pp. 6269, 2010.

[9] R. Landauer "Irreversibility and Heat Generation in the Computational Process", IBM Journal of Research and Development, Vol. 5, No. 3, pp. 183-191, 1961.

[10] C.H. Bennett "Logical Reversibility of Computation", IBM J. Research and Development, Vol 17, No. 6, pp. 525-532, 1973.

[11] M. Nielsen and I. Chuang, "Quantum Computation Quantum Information", Cambridge, U.K. Cambridge Univ. Press, 2000.

[12] R. P. Feynman "Quantum mechanical computers", Foundations of physics, Vol. 16, No. 6, pp. 507-531, 1986.

[13] T. Toffoli "Reversible computing", Springer Berlin Heidelberg, pp. 632-644, 1980.

[14] Fredkin, Edward and T. Toffoli "Conservative logic", Springer London, pp. 47-81, 2002.

[15] M. Haghparast and K. Navi "A novel fault tolerant reversible gate for nanotechnology based systems", American Journal of Applied Sciences, Vol. 5, No, 5, pp. 519, 2008. 
[16] A. N. Bahar, M. Habib and N. K. Biswas "A Novel Presentation of Toffoli Gate in Quantum-dot Cellular Automata (QCA)", International Journal of Computer Applications, Vol. 82, No. 10, pp. 1-4, 2013.

[17] A. N. Bahar, S. Waheed, M. A. Uddin and M.A. Habib "Double Feynman Gate (F2G) in Quantum dot Cellular Automata (QCA)", International Journal of Computer Science Engineering (IJCSE), Vol. 2, No. 6, pp. 351-355, 2013.

[18] M.A. Shafi, M.S. Islam and A.N. Bahar "A Review on Reversible Logic Gates and it's QCA Implementation", International $\mathbf{J}$ of Computer Applications (IJCA), Vol. 128, No. 2, pp. 27-34, 2015.

[19] B. Meurer, D. Heitmann and K. Ploog "Excitation of three dimensional quantum dots", Physical Review, Vol. 68, pp. 1371, 1992.

[20] I. Amlani, A. Orlov, G. Toth, G. H. Bernstein, C. S. Lent and G. L. Snider "Digital Logic Gate Using Quantum-dot Cellular Automata", Science, Vol. 284, No. 5412, pp. 289-291, 1999.
[21] A. Peres "Reversible logic and quantum computers", Physical review A, Vol. 32, No. 6, pp. 3266, 1985.

[22] D. P. Vasudevan, P.K. Lala, J. Di and J.P. Parkerson "Reversible-logic design with online testability", Instrum Meas IEEE Trans Vol. 55, No. 2, pp. 406-414, 2006.

[23] K. Walus, T. J. Dysart, G. A. Jullien and R. A. Budiman "QCADesigner: A rapid design and simulation tool for quantum-dot cellular automata", Transactions on Nanotechnology, IEEE Vol. 3, No. 1, pp. 26-31, 2004.

[24] "QCADesigner" http://www.mina.ubc.ca/qcadesigner [Online; Accessed: 25 October-2015].

[25] A. Sarker, A. N. Bahar, P. K. Biswas and M. Morshed "A novel presentation of peres gate (pg) in quantum-dot cellular automata (QCA)", European Scientific Journal, Vol. 10, No. 21, pp. 101-106, 2014.

[26] A. N. Bahar, S. Waheed and N. Hossain "A new approach of presenting reversible logic gate in nanoscale", SpringerPlus, Vol. 4, No.153, 2015.

[27] R. J. Baker "CMOS: circuit design, layout and simulation", John Wiley and Sons, Vol. 18, 2011. 